\title{
Effect of aligned carbon nanotubes on electrical conductivity behaviour in polycarbonate matrix
}

\author{
M M LARIJANI*，E J KHAMSE ${ }^{\dagger}$ ，Z ASADOLLAHI and M ASADI \\ Nuclear Science and Technology Research Institute (NSTRI), Karaj, Iran \\ ${ }^{\dagger}$ Department of Science, Zanjan University, Zanjan, Iran
}

MS received 24 April 2011; revised 27 July 2011

\begin{abstract}
This article reports effects of alignment of embedded carbon nanotubes in a polycarbonate polymer matrix under magnetic, direct and alternating current electric fields on the electrical properties of the resulting nanocomposites. Composites consisting of different quantities of carbon nanotubes in a polycarbonate matrix have been prepared using a solution casting technique. The effects of field strength and nanotube concentration on the resulted network structure and conductivity of the composites were studied by in situ optical microscopy, transmission electron microscopy and four-point probe technique. The results showed that the composites prepared in the presence of field had better conductivity than those of as-prepared composites. It was also concluded that the application of alternating current electric field and magnetic field in this system led to the formation of relatively continuing networks while direct current electric field only prevented agglomeration of the carbon nanotubes in the polycarbonate matrix and created relatively uniform distribution of nanotubes in the matrix.
\end{abstract}

Keywords. Carbon nanotube; nanocomposite; electrical effect; magnetic effect.

\section{Introduction}

To reinforce materials, commonly nanoparticles are incorporated into host matrices. Among various nanoparticles, carbon nanotubes (CNTs) are the ideal candidates because of their intrinsic properties such as high aspect ratio (length to diameter ratio) and nanoscale diameter. There are currently several methods to process polymer/carbon nanotube composites including melt mixing (Lin et al 2006; Krause et al 2009), in situ polymerization (Park et al 2002; Jiang et al 2005) and solution processing (Kymakis et al 2002) among which the melt mixing method is the preferred one. There are two important points in nanocomposites: (i) uniform dispersion of CNTs in matrix and (ii) orientation and alignment of CNTs in composite (Lin et al 2006; Chandra et al 2007; Oliver et al 2008). It is difficult to obtain uniform dispersion of nanotubes in the matrix because of the existence of high Van der Waals interaction between nanotubes (Pötschke et al 2003). By controlling orientation of nanotubes within the matrix, optimum material properties will be obtained. In order to enhance performance, these processes are desirable that distribute nanotubes randomly or align in a preferred direction in the matrix. In some applications, orientation and alignment of CNTs in specific direction are desirable to obtain optimum results in low fractions of the filler such as light-emitting diode (Ou et al 2009) and increasing of mechanical resistance of the

\footnotetext{
*Author for correspondence (mmojtahedzadeh@nrcam.org)
}

matrix in alignment direction (Wang et al 2008). The magnetic and electric fields are expected to influence the alignment of CNTs in the matrix and in conclusion, to influence the electrical resistivity of the composites. Several techniques have been presented to address this issue. For example, Li et al (2006) reported anisotropic properties of aligned SWNT modified poly (methyl methacrylate) nanocomposites. Wang et al (2008) reported the effects of CNTs alignment on the electrical conductivity and mechanical properties of SWNT/epoxy nanocomposites. They stretched the semi-dried mixture along one direction and showed that electrical conductivity and mechanical properties of the composites improved in the direction of stretch more than the perpendicular one. Camponeschi et al (2007) probed the effects of orientation and alignment of CNTs embedded in an epoxy polymer matrix under the magnetic field on the mechanical properties of the resulting nanocomposite. They also showed that the effect of an external magnetic field on carbon nanotube alignment appears to be dependent upon the polymer matrix used in the composite. Their results showed that the carbon nanotubes appear to have an affinity for polymers with amine groups that are not uniform for all epoxy polymers. Park et al (2008) investigated the strain-dependent electrical resistance characteristics of MWCNT/polymer composites. Their results showed that with increasing the stretch due to the alignment of nanotubes, their resistivity decreased. Martin et al (2005) showed that the formation of CNT network can be induced by the application of an external electric field. They used direct current (d.c.) and alternating current (a.c.) electric fields and found that a.c. field was more effective than d.c. one. 
The aim of this paper is to investigate influence of magnetic, d.c. and a.c. electric fields on the electrical resistivity of the polycarbonate (PC)/carbon nanotube composites and to evaluate their effects on the improvement of electrical conductivity of PC/MWCNT composites.

\section{Experimental}

MWCNT (diameter, 10-20 nm; length, 5-15 $\mu \mathrm{m}$ ) were purchased from Ionic Technology Inc (Germany) with a purity of higher than $95 \%$. Energy-dispersive X-ray spectroscopy (EDS) technique used for the elemental analysis identified $\mathrm{Fe}$ and $\mathrm{Ni}$ as catalyst particles in the purchased CNTs. For evaluating the effect of magnetic field on the CNT alignment, the solution of nanotubes and hexane (to prevent damage of MWCNTs structure) was ball milled at $150 \mathrm{rpm}$ for $5 \mathrm{~min}$. It was expected that ball milling process could separate sticking CNTs. The detailed preparation conditions are listed in table 1. At first, certain amounts of PC pellets were dissolved in chloroform by stirring for $15-20 \mathrm{~min}$ at $50^{\circ} \mathrm{C}$. MWCNTs with concentrations in the range of $0.05-0.7 \mathrm{wt} \%$ were dispersed in chloroform via sonicator THYRISTOR 600 for $30 \mathrm{~min}$. The sonication power was held low to prevent damage of MWCNTs structure. PC/MWCNT/chloroform mixture was stirred and heated up to about $50^{\circ} \mathrm{C}$ for $5-10 \mathrm{~min}$, then the prepared solutions were put into glass dishes of 6 diameters in 2 series. Some of them were prepared without magnetic field, whereas the only one in the presence of magnetic field, $0.112 \mathrm{~T}$ (chosen because of technical limit). The films were obtained after drying at room temperature.

Harish (2005) showed more homogeneous solution of filler in mixture of two solvents at low CNT concentration. Based on the literature review, toluene is one of the most dominant solvents which provide good solubility for CNT while chloroform is the proper solvent for PC. Since toluene destroyed polycarbonate structure, we dispersed nanotubes in two steps. First, the various dilute MWCNT solutions, typically around $0.012,0.025,0.050$ and $0.1 \mathrm{wt} \%$ in toluene, were prepared by using ultrasonication for about $30 \mathrm{~min}$. It was reported that a better alignment of CNTs was achieved in presence of electric fields at low fraction of CNTs (Steinert and Dean 2009). In order to disperse CNTs uniformly in composites after removing toluene in oven, the solution of MWCNT and chloroform was sonicated for about 30 min. The sonicated CNTs were mixed with dissolved

Table 1. Experimental details of composites synthesis.

\begin{tabular}{lc}
\hline Sample & Method of preparation \\
\hline$A$ & As-prepared sample \\
$B$ & Series of samples supported by \\
& $0 \cdot 112 \mathrm{~T}$ magnetic field \\
$C$ & Series of samples supported by 1.5 and \\
$D$ & $3 \mathrm{kV}$, a.c. electric field \\
& Series of samples supported by 1.5 and \\
& $3 \mathrm{kV}$, d.c. electric field \\
\hline
\end{tabular}

PC and stirred in a mixture. The solutions were cast on glass dishes and were put in a.c. and d.c. electric fields $(\mathrm{V}=1.5,3 \mathrm{kV})$ created between two plaques at a distance of $100 \mathrm{~mm}$ and the solvent was left to evaporate slowly. Typical thickness of the composite samples was $\sim 20 \mu \mathrm{m}$.

The electrical conductivity was measured at room temperature by a four-point probe technique using an electrometer (GTM unito) with two outer current probes and two inner voltage ones. The composite surface was coated with four rod-shaped copper layers $\left(2 \times 14 \mathrm{~mm}^{2}\right)$ separated from each other by $2 \mathrm{~mm}$ using magnetron sputtering method. The applied voltage ranged within $0 \cdot 02-500$ Vs. Four copper wires were used to establish the connection between the coated copper layers with GTM probes. The sheet conductivity of PC/MWCNT composites was evaluated by

$$
\sigma^{-1}=(V / I) \pi t / \operatorname{Ln} 2,
$$

where $t$ and $s$ are thickness of the sheet $(20 \mu \mathrm{m})$ and typical probe spacing $(s \sim 2 \mathrm{~mm}), V$ and $I$ are the applied voltage between inner probes and the measured current between outer probes, respectively.

Scanning electron microscopy (SEM) images of sample cross-section were obtained using an LEO 440i. To observe alignment of CNTs in the composite and investigate the degree of alignment, in situ optical microscopy (OM) and transmission electron microscopy (TEM) were used. The samples for TEM were prepared by ultramicrotomy with a cutting thickness of $60 \mathrm{~nm}$. TEM was conducted using Philips EM208S at $100 \mathrm{kV}$ and optical images were obtained from Dino/Lite digital microscope.

\section{Results and discussion}

Figure 1 shows OM images of as-prepared and magneticprepared composites with $0.5 \mathrm{wt} \%$ CNTs at $300 \times$ magnification. In these pictures the white spots are due to the light reflection of the microscope on the composite. From figure $1 \mathrm{a}$, one observes a random distribution of agglomerated CNTs in the as-prepared sample (A), while a slight alignment is achieved in the presence of the magnetic field. Due to magnetic susceptibility of CNTs (Heremans et al 1994), it should be possible to align them along the magnetic field direction. In fact, CNTs have a strong structural anisotropy which causes possibility of the alignment of them along the direction of an applied magnetic field via utilization of this strong anisotropy diamagnetic property. Camponeschi et al (2007) have shown that CNT filler alignment can occur at high magnetic fields while in low magnetic fields they result in a separated and oriented CNT. According to their results, we think that in our case, the field strength is probably insufficient to perfectly orient nanotubes against viscosity of the polymeric solution along the field direction. Figure 2 shows TEM image of sample B with $0.1 \mathrm{wt} \%$ CNTs. The dark spots in the image are related to the catalyst particles. Due to high magnification, it is difficult to observe a large number of nanotubes within the visual field of TEM. Therefore, 


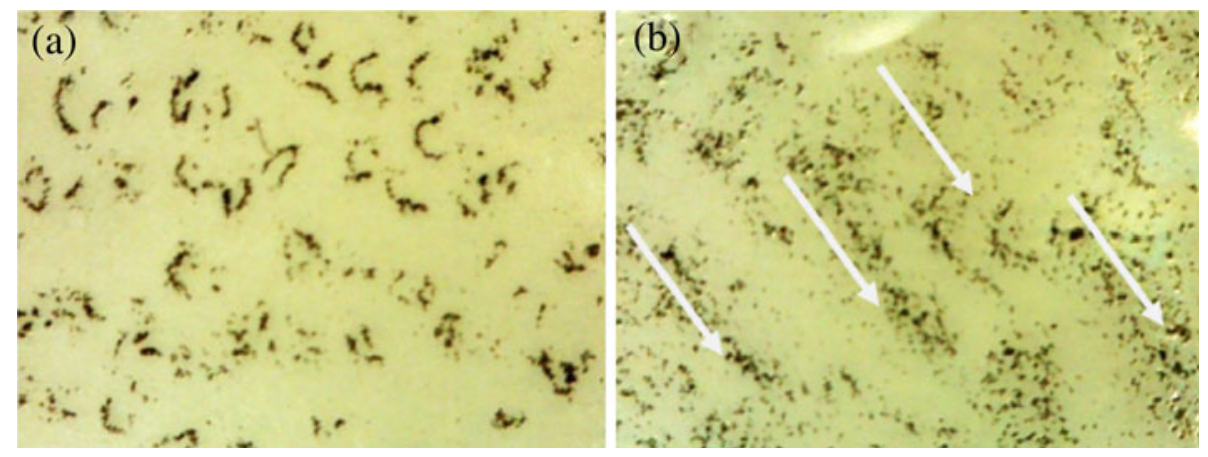

Figure 1. Optical microscopy images of a. as-prepared sample and $\mathbf{b}$. sample in presence of magnetic field with $0.5 \mathrm{wt} \%$ nanotubes $(300 \times)$. Arrows show direction of aligned nanotubes in polycarbonate composite.

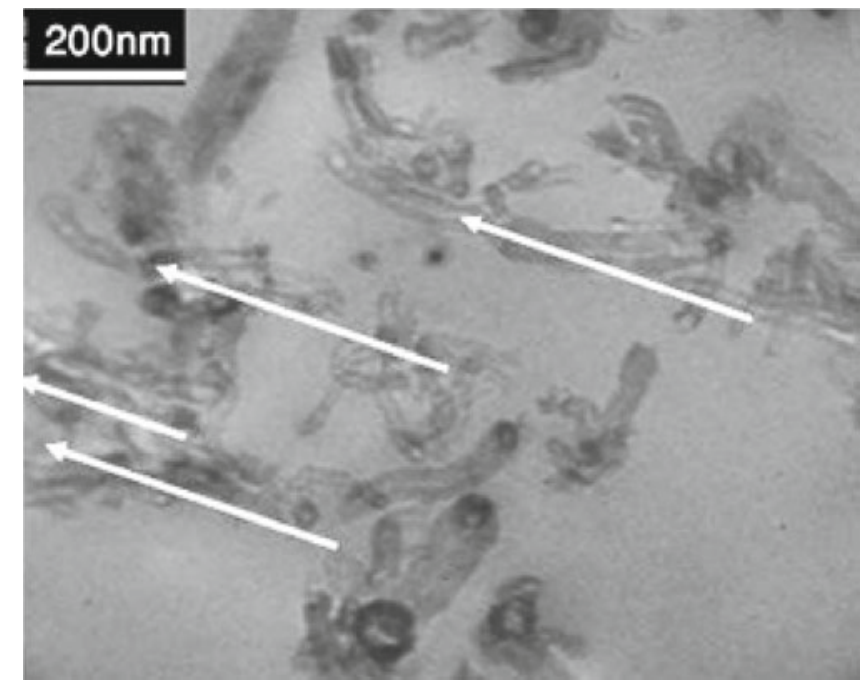

Figure 2. TEM image of polycarbonate/carbon nanotube composite containing $0 \cdot 1 \mathrm{wt} \%$ nanotubes loading fraction in magnetic field strength of $0 \cdot 112 \mathrm{~T}$.

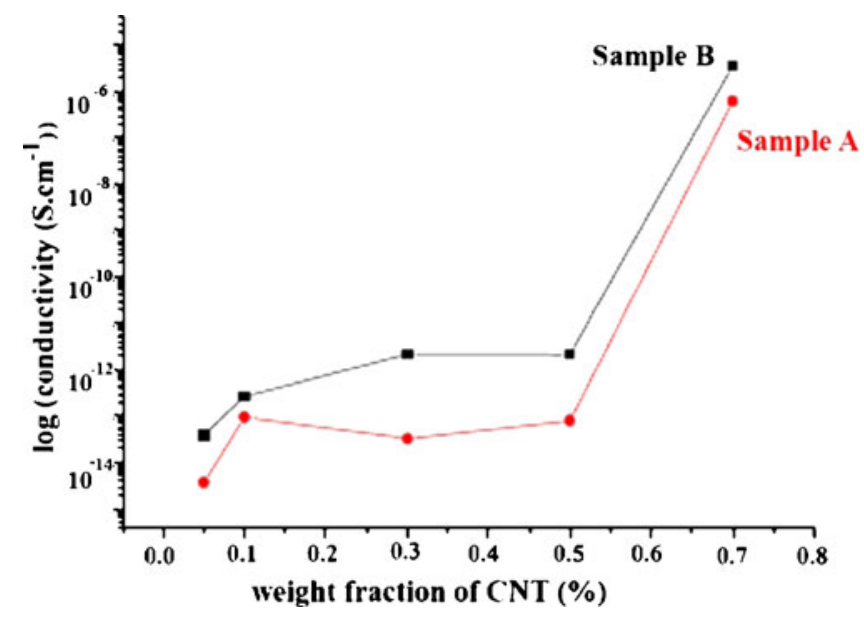

Figure 3. Comparison of electrical conductivity of polycarbonate/carbon nanotube composites in magnetic field strength of $0 \cdot 112 \mathrm{~T}$ and as-prepared samples as a function of nanotube weight fraction. discussing about alignment of CNTs with respect to low strength of field will be difficult. Figure 3 presents the logarithmic conductivity results of MWCNT/PC composites as a function of weight fraction of MWCNTs. One can divide both plots into three regions. In region 1, with increasing CNT content up to $0 \cdot 1 \mathrm{wt} \%$ conductivity increases and afterwards approximately flattens in the range of $0.1-0.5 \mathrm{wt} \%$ (region 2) (Ounaies et al 2003). An excess CNT concentration from the above values causes a sharp increase in conductivity (region 3 ). Comparison of samples $A$ and $B$ shows that at low concentrations, the difference between conductivity for both samples is minor. However, the difference increases from 0.1 to $0.5 \mathrm{wt} \%$, indicating the effectiveness of magnetic field in increasing the conductivity of composites in this range of CNT content. At even higher concentrations, the conductivity increases from $\sim 10^{-13}$ to $10^{-7} \mathrm{~S} / \mathrm{cm}$ in sample $A$ and from $10^{-11}$ to $10^{-5} \mathrm{~S} / \mathrm{cm}$ in sample $B$, indicating $10^{6}$ order of magnitude improvement in electrical conductivity in both cases. The approximate percolation threshold of two samples was found to be around $0.5 \mathrm{wt} \%$ CNT loads. Complied with percolation theory (Stauffer and Aharony 2003), percolation threshold is a critical value which is due to formation of the conductive path. At this value, conversion of insulator to conductor will occur. It is worth knowing that the presence of magnetic particles such as Fe and Ni could also increase more the effect of magnetic field because the repulsion force between the magnetic particles located in or on nanotubes improves further the dispersion of oriented CNTs in the polymer matrix. Increase of conductivity is certainly a consequence of CNT alignment in composite during evaporation. At low concentrations (region 1) with the increase of CNT content, the conductor sites increase in matrix for electron hopping mechanism. In region 2 , increase of conductivity is impeded by increase of CNT content. In this region, the Van der Waals interaction causes agglomeration of CNTs and leads to a flattened region in the plot. In fact, this plateau region is a compromise between electron hopping effect and agglomeration of CNTs that is a consequence of increasing of CNT content. The sharp increase of conductivity in region 3 is probably due to contact between thin polymer coated or uncoated CNTs which leads to increasing of path numbers 

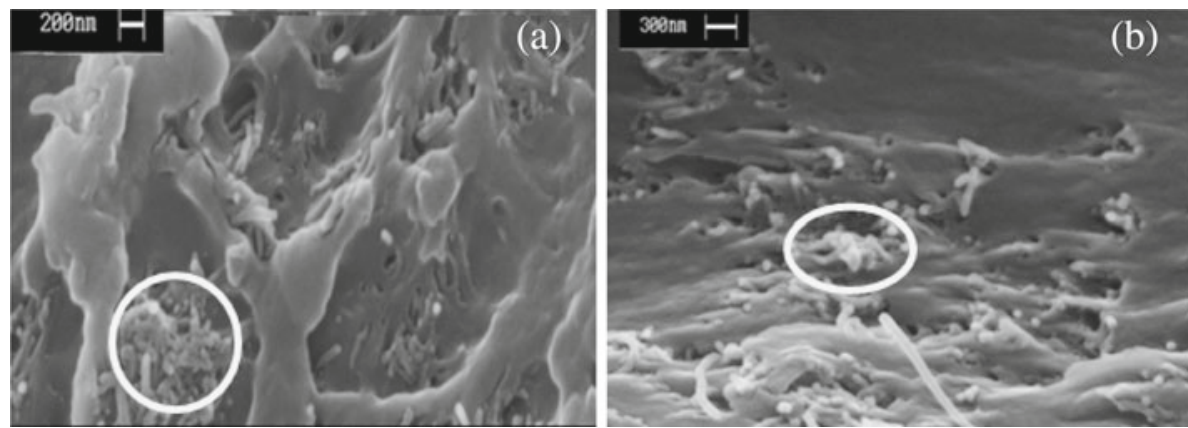

Figure 4. SEM images of cross section of $\mathbf{a}$. as-prepared sample and $\mathbf{b}$. sample prepared in presence of magnetic field with $0.3 \mathrm{wt} \%$ nanotubes. (Circles show nanotube bundles).

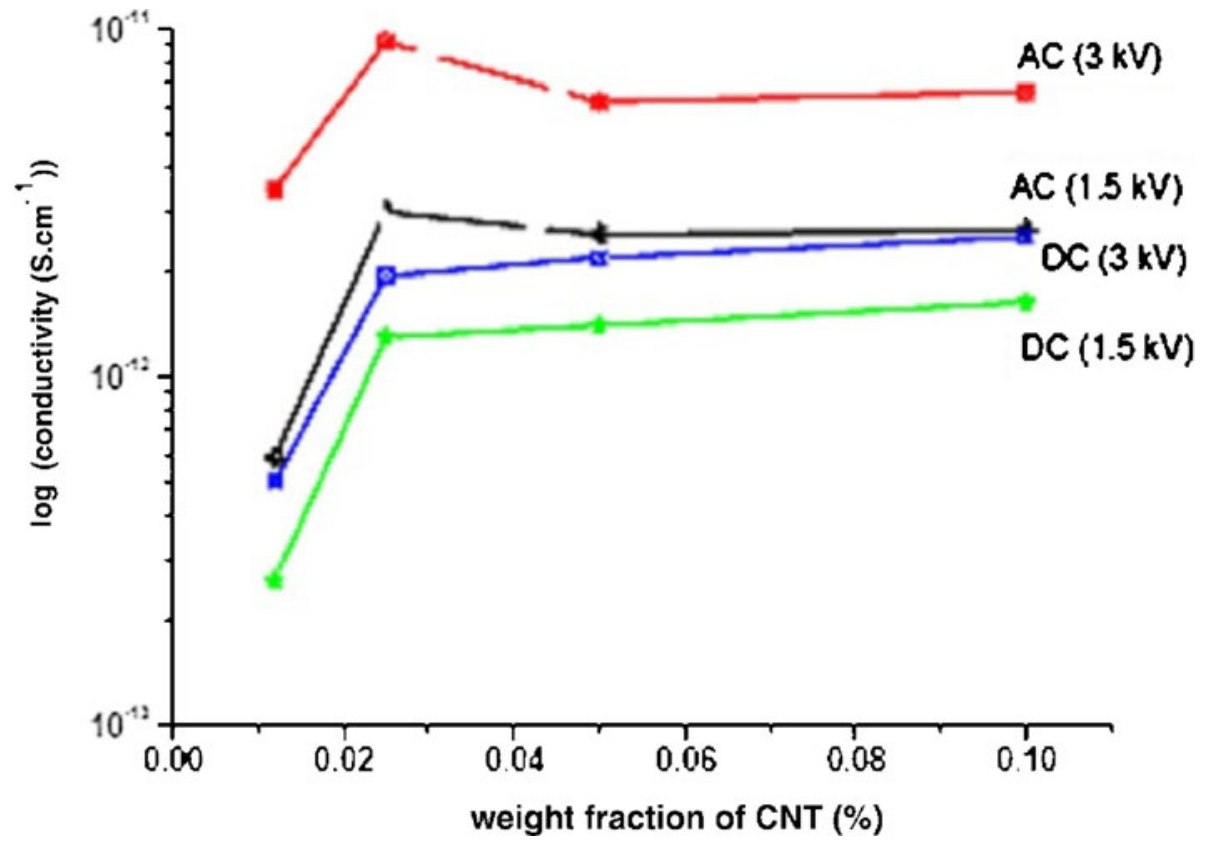

Figure 5. Comparison of electrical conductivity of polycarbonate/carbon nanotube composites in alternate and direct current electric fields of 1.5 and $3 \mathrm{kV}$ as a function of nanotube weight fraction.
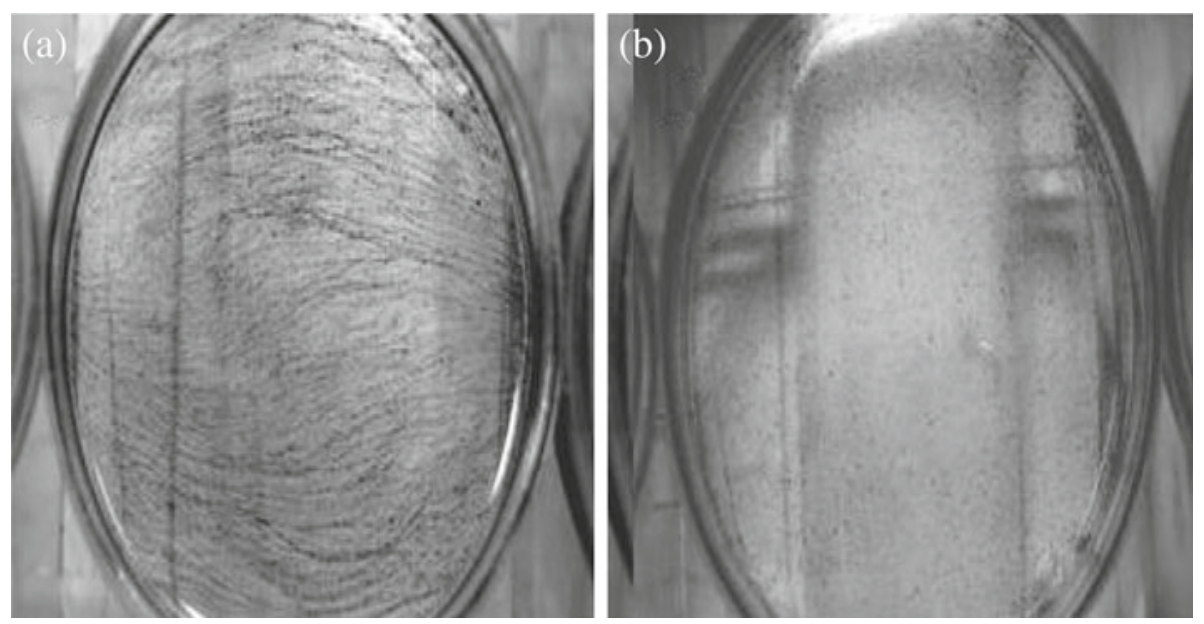

Figure 6. Macro photos of films in a. alternate and $\mathbf{b}$. direct current electric fields of $3 \mathrm{kV}$. 
between opposite sites and formation of CNT networks. The conductivity of sample $B$ is more than sample $A$ because of less agglomeration of nanotubes due to the existence of the field. Agglomeration of nanotubes in the matrix causes a large distance between the conductive sites for electrons hopping which decreases electrical conductivity. In contrast, it is thought that due to more junctions creation between nanotubes the alignment increases electrical conductivity of the composite that results in the formation of more conducting networks in the polymer. Figure 4 compares the dispersion quality between samples $A$ and $B$ with 0.3 wt $\%$ CNTs. The repulsion force in sample $B$ between similar poles of magnetic particles prevents their aggregation and causes better dispersion in the polymer matrix (figure $4 \mathrm{~b}$ ), while in sample $A$, MWCNTs form more bundles under the same processing conditions. As shown in figure 2 the degree of alignment is low at $0.112 \mathrm{~T}$. The reasons seem to be low strength of the magnetic field, the repulsion force between CNTs and the viscosity of the polymeric solution. While the formation of the CNTs network is not impeded by imperfect alignment of nanotubes in high concentrations.

Figure 5 shows effect of electric field applied to $\mathrm{PC} / \mathrm{MWCNT/chloroform} \mathrm{solutions} \mathrm{during} \mathrm{sample} \mathrm{prepara-}$ tion. It is clear that the type and strength of electric field play an important role on the composite conductivity. In the d.c. or a.c. fields, with the increase of voltage the conductivity increases but a.c. field is more effective than d.c. one. In all cases due to the incorporation of conducting sites in the insulator polymer matrix which causes easier electrons hopping, conductivity up to $0.02 \mathrm{wt} \%$ CNTs content increases. Afterwards, the plateau behaviour is observed because of nanotubes agglomeration which leads to a stable distance between conductive sites in the matrix. This stability is a consequence of low CNT concentration. The a.c.
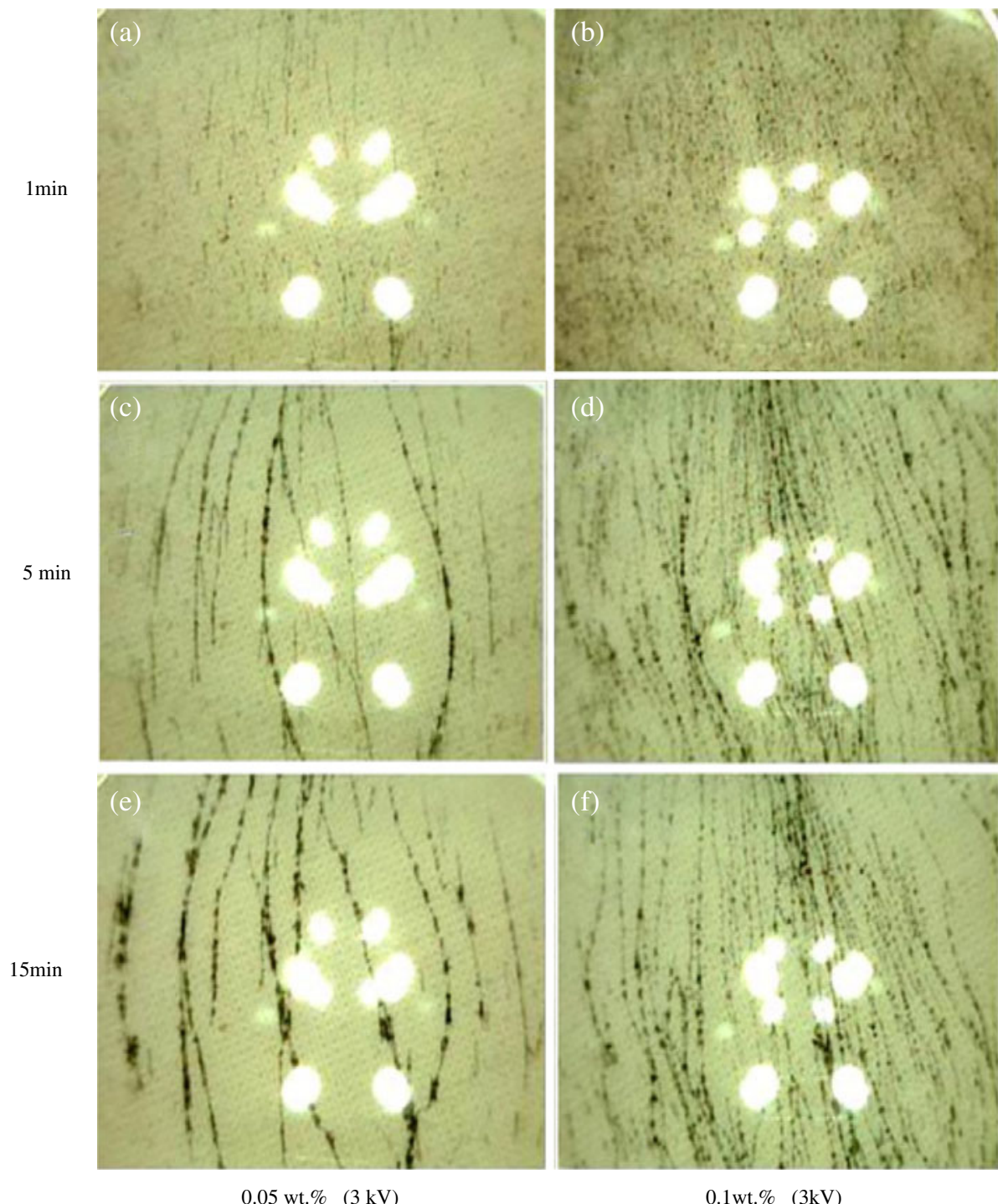

Figure 7. In situ optical microscopy images of samples prepared in presence of altering electric field (a, c, e) and (b, d, f) containing 0.05 and 0.01 wt\% CNT loading, respectively during $15 \min (300 \times)$. 


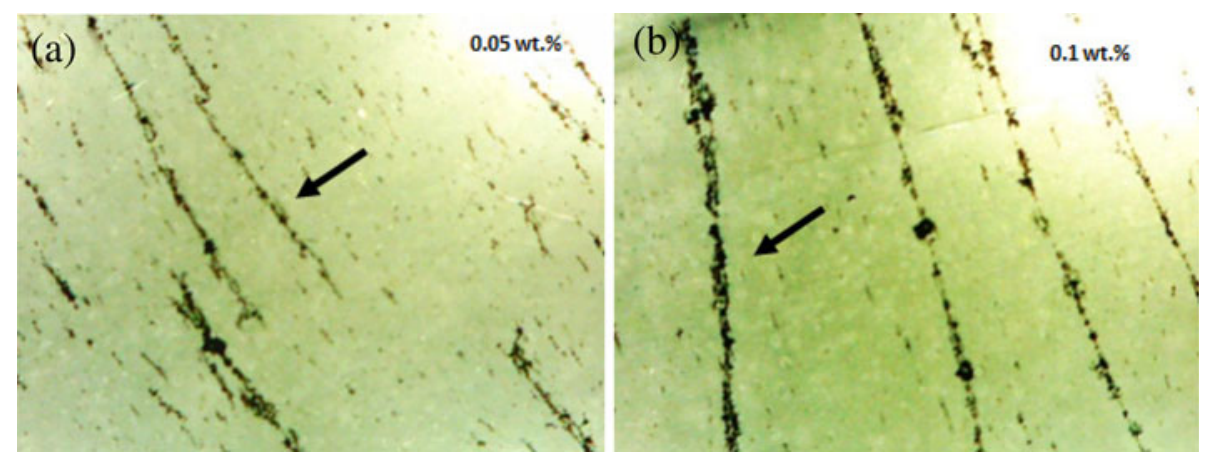

Figure 8. Carbon nanotube network formed in $3 \mathrm{kV}$ alternating electric field with a. 0.05 wt. $\%$ and b. 0.1 wt. $\%$ nanotubes after $15 \mathrm{~min}(500 \times)$. White areas are light of source.
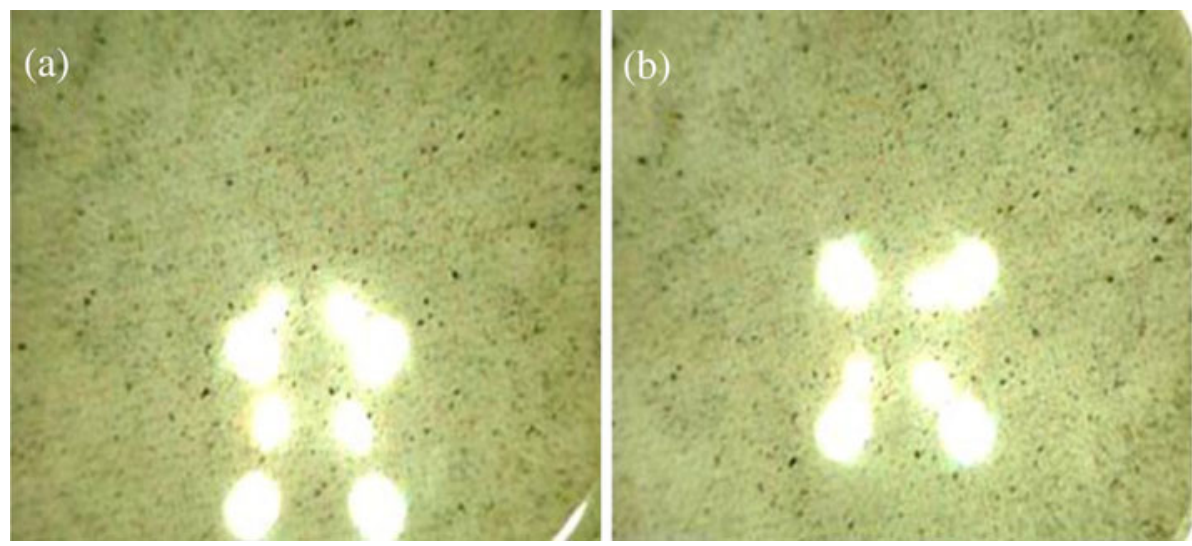

Figure 9. In situ optical microscopy images of samples prepared in presence of direct current (d.c.) electric field with $0.05 \mathrm{wt} . \%$ nanotube concentration after a. $1 \mathrm{~min}$ and b. $180 \mathrm{~min}$ at $3 \mathrm{kV}$ d.c. field $(300 \times)$. White areas are light of source.

electric field induces dipole moments in MWCNTs and produces translational and rotational forces which causes alignment of MWCNTs in the direction of the applied field and also prevents their agglomeration (Ma et al 2008). In other words, CNTs near both the electrodes were rotated, oriented and moved toward the nearest electrode. These CNTs then became sources of high field strength and locations for the connection of other nanotubes. Rotation and alignment of CNTs became connected to one another, resulting in networks of uniform connection along the direction of the electric field. Of course, high matrix viscosity resists against their movement. It is important to note that the connection of the networks did not vary with distance from both electrodes. Increasing the strength of a.c. electric field leads to the increase of induced forces on CNTs and results in nanotubes conducting channels which consequently enhance the electrical conductivity of the composite whereas in d.c. one, due to the wide range, Coulomb force prevents the agglomeration of nanotubes and as a result better distribution of them is achieved. Figure 6 shows macrophotos of the composites containing $0.1 \mathrm{wt} \% \mathrm{CNT}$ during preparation in a.c. and d.c. fields of $3 \mathrm{kV}$. These figures show the formation of network of CNTs in a.c. field (figure 6a) and random distribution of fillers in d.c. one (figure 6b). From this figure, it is obvious that a lot of CNT conducting networks are formed in a.c. field whereas in d.c. field, CNTs are distributed randomly in composite.

Figure 7 shows composites with $0.05 \mathrm{wt} \%$ and $0.1 \mathrm{wt} \%$ CNT contents under $3 \mathrm{kV}$ a.c. electric field over time. In the presence of a.c. field, the growth of network starts from both electrodes and since the poles change alternatively, a straight line of CNTs is created. The formation of network in a.c. field starts only a few seconds after applying the electric field and the position of the aligned MWCNTs are not stable over time. In accordance with these figures the neighbouring CNTs start to move toward each other and form thicker line over time. With the increase of carbon nanotube loading fraction, the onset of network formation occurs earlier. Also increasing CNT load increases the number of networks and decreases the number of dead paths (figure 8). White areas are light of the source. As shown in figure 9 even after 180 min of composite preparation no network is formed in d.c. field. It may be due to the low strength of the field, large size of the samples in comparison with other studies 
(Martin et al 2005; Takahashi et al 2006), but because of the wide range, Coulomb forced agglomeration of CNTs is prevented and better dispersion is obtained.

\section{Conclusions}

Carbon nanotubes were used as filler in polycarbonate matrix. Composites were prepared using solution casting method. It was demonstrated that the application of a.c. electric field and magnetic field in this system led to the formation of relatively continuing networks and the applied d.c. electric field only prevented agglomeration of CNTs. Due to the formation of the network of CNTs in a.c. electric field, PC/MWCNTs composites became more conductive in a.c. field than in d.c. one.

\section{References}

Camponeschi E, Vance R, Al-Haik M, Garmestani H and Tannenbaum R 2007 Carbon 452037

Chandra A, Kramschuster A J, Hu X and Turng L S 2007 Effect of injection molding parameters on the electrical conductivity of polycarbonate/carbon nanotube nanocomposites, Proc. of ANTEC, p. 2184

Harish M 2005 Processing and study of carbon nanotube/polymer nanocomposites and polymer electrolyte materials, $\mathrm{PhD}$ Thesis, Indian Institute of Technology, Madras
Heremans J, Olk C H and Morelli D T 1994 Phys. Rev. B49 15122 Jiang X, Bin Y and Matsau M 2005 Polymer 467418

Krause B, Pötschke P and Häubler L 2009 Compos. Sci. Technol. 691505

Kymakis E, Alexandou I and Amaratunga G A J 2002 Synth. Met. 12759

Li W, Wang Q and Dai J 2006 Bull. Mater. Sci. 29313

Lin B, Sundaraj U and Pötschke P 2006 Macromol. Mater. Eng. 291 227

Ma C, Zhang W, Ji L, Zhang R, Koratkar N and Liang J 2008 Carbon $\mathbf{4 6} 706$

Martin C A, Sandler J K W, Windle A H, Schwarz M K, Bauhofer W, Schulte K and Schaffer M S P 2005 Polymer 46877

Oliver A, Bult J, Le Q V, Mbaruku A L and Schwartz J 2008 Nanotechnology 19505702

Ou E C W et al 2009 Acs Nano 32258

Ounaies Z, Park C, Wise K E, Siochi E J and Harrison J S 2003 Compos. Sci. Technol. 631637

Park C et al 2002 Chem. Phys. Lett. 364303

Park M, Kim H and Youngblood J P 2008 Nanotechnology 19 055705

Pötschke P, Bhattacharyya A R, Janke A and Georing H 2003 Compos. Interf. 10389

Stauffer D and Aharony A 2003 Introduction to percolation theory (London: Taylor \& Francis)

Steinert B W and Dean D R 2009 Polymer 50898

Takahashi T, Murayama T, Higuchi A, Awano H and Yonetake K 2006 Carbon 441180

Wang Q, Dai J, Li W, Wei Z and Jiang J 2008 Compos. Sci. Technol. 681644 